\title{
Effects of dietary gelatin hydrolysates on bone mineral density in magnesium- deficient rats
}

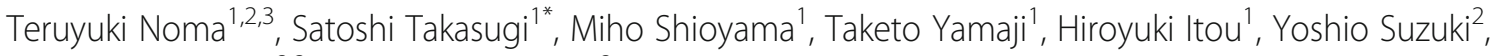
Keishoku Sakuraba ${ }^{2,3}$ and Keisuke Sawaki ${ }^{2}$

\begin{abstract}
Background: The major types of commercially available gelatin hydrolysates are prepared from mammals or fish. Dietary gelatin hydrolysates from mammals were reported to improve bone mineral density (BMD) in some animal models. In contrast, there is limited study showing the effects of dietary gelatin hydrolysates from fish on BMD. The quantity and structure of peptides in the plasma after oral administration of gelatin hydrolysates depend on the gelatin source, which suggests that the biological activity of gelatin hydrolysates depend on the gelatin source. This study examined the effects of fish-derived gelatin hydrolysate (FGH) or porcine-derived gelatin hydrolysate (PGH) intake on BMD and intrinsic biomechanical properties in magnesium (Mg)-deficient rats as a model showing the decrease in both BMD and intrinsic biomechanical properties.
\end{abstract}

Methods: Four-week-old male Wistar rats were assigned into four groups: a normal group was fed a normal diet (48 mg Mg/100 g diet), a Mg-deficient (MgD) group was fed a MgD diet (7 mg Mg/100 g diet), a FGH group was fed a MgD + FGH diet (5\% FGH), and a PGH group was fed a MgD + PGH diet (5\% PGH) for 8 weeks. At the end of the study, BMD and intrinsic biomechanical properties of the femur were measured.

Results: The MgD group showed significantly lower Young's modulus, an intrinsic biomechanical property, and trabecular BMD of the femur than the normal group; however, the MgD diet did not affect cortical BMD and cortical thickness. Both the FGH and the PGH groups showed significantly higher cortical thickness and ultimate displacement of the femur than the normal group, but neither type of gelatin hydrolysate affected Young's modulus. Furthermore, the FGH group, but not the PGH group, showed significantly higher trabecular BMD than the MgD group.

Conclusions: This study indicates that FGH and PGH increase cortical thickness but only FGH prevents the decrease in trabecular BMD seen in Mg-deficient rats, while neither type of gelatin hydrolysate affect intrinsic biomechanical properties.

Keywords: Collagen, Magnesium deficiency, Bone mineral density, Cortical bone, Trabecular bone, Rats, Peptide

\section{Background}

Osteoporosis is a multifactorial bone disease, featured by low bone mineral density and microarchitectural deterioration of bone tissue, resulting in loss of mechanical strength and increased risk of fractures [1]. While calcium is the most well-known mineral for the prevention of osteoporosis, other minerals such as zinc, iron, and

\footnotetext{
*Correspondence: satoshi.takasugi@meiji.com

'Division of Research and Development, Food Science Research Laboratories,

Meiji Co., Ltd., 540 Naruda, Odawara, Kanagawa 250-0862, Japan

Full list of author information is available at the end of the article
}

magnesium $(\mathrm{Mg})$ also play an important role in bone metabolism. Magnesium is one of the nutrients most likely to be consumed at levels below the recommended daily allowance (RDA) [2]. Some cross-sectional studies have demonstrated that dietary $\mathrm{Mg}$ was positively correlated with bone mineral density (BMD) in elderly subjects [3] and middle-aged women [4], and a longitudinal study have also showed that a greater intake of $\mathrm{Mg}$ was associated with less of a decline in BMD in elderly subjects [3]. In rats, $\mathrm{Mg}$ deficiency was reported to reduce both BMD [5] and intrinsic biomechanical properties [6]. 
Collagen is a major component of connective tissues, such as bone, dermis, cartilage and tendons. Gelatin, a denatured collagen, is produced mainly from swine, fish, and birds. Gelatin hydrolysate is produced by the hydrolysis of gelatin, and the major types of commercially available gelatin hydrolysates are prepared from swine or fish. Some researchers reported that dietary gelatin or gelatin hydrolysates from mammals improve BMD in ovariectomized mice [7], growing rats [8], calciumdeficient rats [8], and low protein-fed rats [9]. In contrast, there is limited study showing the effects of dietary gelatin hydrolysates from fish on BMD [10].

Hydroxyproline (Hyp) is a major component of collagen. In vitro studies have shown that Hyp-containing peptides or gelatin hydrolysate-derived peptides have biological activity, including chemotactic activity for neutrophils, fibroblasts, [11, 12] and monocytes [13], as well as inhibitory effects on the angiotensin-converting enzyme [14, 15]. Ohara et al. [16] compared the structure and amount of Hyp-containing peptides in human plasma after peroral administration of fish-derived gelatin hydrolysates $(\mathrm{FGH})$ or porcine skin-derived gelatin hydrolysates (PGH), and showed that the structure and amount of peptides in human plasma after peroral administration of gelatin hydrolysates depend on the gelatin source, and that alanine- or glycine-containing peptides were detected only in the human administered with FGH. These facts suggest that the biological activity of gelatin hydrolysates depend on the gelatin source.

This study aimed to investigate the effects of FGH and PGH intake on BMD and intrinsic biomechanical properties in $\mathrm{Mg}$-deficient $(\mathrm{MgD})$ rats as a model showing the decrease in both BMD and intrinsic biomechanical properties.

\section{Methods}

\section{Diets}

FGH and PGH were kindly provided by Nitta Gelatin (Osaka, Japan). Mean molecular weight of these gelatin hydrolysates was $5000 \mathrm{Da}$. We employed AIN-76 as the normal diet (48 mg Mg/100 g diet). The MgD diet contained $7 \mathrm{mg} \mathrm{Mg} / 100 \mathrm{~g}$ diet. For $\mathrm{MgD}+\mathrm{FGH}$ and $\mathrm{MgD}+\mathrm{PGH}$ diets, the $5 \%$ casein in the MgD diet was replaced with FGH and PGH, respectively. The composition of each diet and analysis values of calcium, phosphorus, and $\mathrm{Mg}$ are detailed in Table 1.

\section{Animals}

This research was approved by the Institutional Animal Care and Use Committee (IACUC) board of the Meiji Co., Ltd. (Ethics approval code: No. 2015_3871_0088). Twenty-four, 3-week-old male Wistar rats (Japan SLC, Inc.,Shizuoka, Japan) were reared according to the Meiji Co., Ltd. ethics committee guidelines on animal use. The
Table 1 Composition of the experimental diets

\begin{tabular}{lllll}
\hline Ingredients (\%) & Normal & MgD & FGH & PGH \\
\hline Casein & 20.0 & 20.0 & 15.0 & 15.0 \\
Fish scale gelatin hydrolysate & 0.0 & 0.0 & 5.0 & 0.0 \\
Porcine skin gelatin hydrolysate & 0.0 & 0.0 & 0.0 & 5.0 \\
DL-methionine & 0.3 & 0.3 & 0.3 & 0.3 \\
Corn starch & 15.0 & 15.0 & 15.0 & 15.0 \\
Sucrose & 50.0 & 50.0 & 50.0 & 50.0 \\
Corn oil & 5.0 & 5.0 & 5.0 & 5.0 \\
Cellulose powder & 5.0 & 5.0 & 5.0 & 5.0 \\
AlN-76 mineral premix & 3.5 & 0.0 & 0.0 & 0.0 \\
AlN-76 mineral premix without magnesium & 0.0 & 3.5 & 3.5 & 3.5 \\
AlN-76 vitamin mix & 1.0 & 1.0 & 1.0 & 1.0 \\
Choline bitartrate & 0.2 & 0.2 & 0.2 & 0.2 \\
Analysis values (mg/100 g) & & & & \\
Calcium & 533 & 530 & 524 & 521 \\
Phosphorus & 553 & 563 & 519 & 519 \\
Magnesium & 48 & 7 & 7 & 7 \\
\hline
\end{tabular}

animals were housed in individual stainless steel cages in an environmentally controlled room $\left(21 \pm 2{ }^{\circ} \mathrm{C}, 55 \pm 15 \%\right.$ humidity, 12-h light/dark cycle). After 4 days of adaptation, animals were assigned into four weight-matched groups of six rats each: a normal group, an $\mathrm{MgD}$ group, a FGH group, and a PGH group. All groups were fed their respective experimental diets and ultraviolet sterilized water ad libitum for 8 weeks. Food intake and body weight were measured weekly. Food efficiency was calculated using the following formula:

Food efficiency = body weight gain/food intake.

At the end of the experimental period, we obtained blood samples from the abdominal aorta under anesthesia with a medetomidine-midazolam-butorphanol mixture [17]. All rats were euthanized by exsanguination through the aorta under the anesthesia. The serum samples were separated by centrifugation at $3000 \times \mathrm{g}$ for $15 \mathrm{~min}$ at $4{ }^{\circ} \mathrm{C}$ and preserved at $-80{ }^{\circ} \mathrm{C}$ until analysis. The both sides of femurs were excised after euthanasia. The left femurs were wrapped in saline-wet gauze and stored at $-20{ }^{\circ} \mathrm{C}$ until mechanical testing as previously described [18]. The right femurs were preserved in a $70 \%$ solution of ethanol (Wako Pure Chemical Industries, Osaka, Japan) for X-ray computed tomography (CT) analysis.

\section{Bone parameters by X-ray CT analysis}

The whole right femur was scanned using the experimental animal CT system (LaTheta LCT-100 M; ALOKA, Tokyo, Japan). Contiguous 1.0-mm slices of the 
whole femur were employed for quantitative measurement. Total, cortical, and trabecular BMDs, and cortical thickness (Ct.Th) of the whole femur were measured by LaTheta software (Version 1.31). Ct.Th of the whole femur was calculated as the mean of all slices. Ct.Th of each slice was calculated using the following formula:

$$
\mathrm{Ct} . \mathrm{Th}=\text { cortical area/cortical center line length } .
$$

\section{Mechanical testing}

We performed a three-point bending test by a mechanical testing system (Bone Strength Tester, model TK252C; Muromachi Kikai, Tokyo, Japan) on the left femur according to the modified methods previously described $[19,20]$. After placing the femur in a $37^{\circ} \mathrm{C}$ saline bath, it was set on a supporter with two loading points $14 \mathrm{~mm}$ apart (the span of the specimen: L). A vertical breaking load was applied to the midpoint between the lower supports by the crosshead at a constant speed $(2.5 \mathrm{~mm} /$ min) until failure occurred. The capacity of the load cell in this test system is $500 \mathrm{~N}$. The work to failure, stiffness, ultimate displacement (d), and ultimate force (F) of the femur were calculated from the load-deformation curve. After failure, the cross-sectional area of the femur was calculated as a hollow ellipse [18, 21, 22]. The major and minor internal and external axes of the crosssectional area were measured by digital caliper (DT-200; Niigata Seiki, Niigata, Japan). Ultimate stress and Young's modulus were calculated as follows:

$$
\begin{gathered}
\text { Ultimate stress }=8 \mathrm{bFL} /\left(a b^{3}-\mathrm{a}^{\prime} \mathrm{b}^{\prime 3}\right) \pi \\
\text { Young's modulus }=4 \mathrm{FL}^{3} / 3 \mathrm{~d}\left(a \mathrm{ab}^{3}-\mathrm{a}^{\prime} \mathrm{b}^{\prime 3}\right) \pi
\end{gathered}
$$

where $\mathrm{a}$ and $\mathrm{b}$ are the major and minor external axes, respectively, and a' and 'b' are the major and minor internal axes, respectively. The work to failure, stiffness, ultimate displacement, and ultimate force represent extrinsic biomechanical properties [23]. Ultimate stress and Young's modulus represent intrinsic biomechanical properties. The work to failure reflects the absorbed energy of specimen until failure occurred. Increased brittleness reduces the work to failure. The stiffness reflects the resistance to elastic deformation, i.e., the structural rigidity of bone. The reciprocal of the ultimate displacement can estimate brittleness [23]. The ultimate force characterizes the strength of a bone.

\section{Biochemical analysis}

Serum levels of total osteocalcin (OC) were assayed using the Rat Gla/Glu-Osteocalcin High Sensitive EIA Set (Takara Bio Inc., Shiga, Japan). Total OC was calculated as the sum of carboxylated OC and undercarboxylated OC. Serum C-terminal cross-linked telopeptides of type I collagen (CTX) and tartrate-resistant acid phosphatase 5b (TRACP5b) were measured by the RatLaps EIA kit and RatTRAP Assay kit, respectively, both of which were manufactured by Immunodiagnostic Systems Nordic A/S (Herlev, Denmark). Resorption index was calculated as the ratio of CTX/TRACP5b [24]. Serum $\mathrm{Mg}$ levels and alkaline phosphatase (ALP) activity were colorimetrically assayed by commercial kits (Wako Pure Chemical Industries, Osaka, Japan).

\section{Statistics}

Data are expressed as mean \pm standard errors. We performed Bartlett's test to determine the homogeneity of variances. Treatment effects were analyzed by one-way ANOVA followed by the Tukey-Kramer test (homogenous variances) or the Kruskal-Wallis test followed by the Steel-Dwass multiple comparison test (heterogenous variances). $P$ values $<0.05$ were considered significant. Additionally, we presented the minimum sample sizes to detect group differences between the $\mathrm{MgD}$ group and the FGH groups for BMD and trabecular BMD in the present study. We employed the Ekuseru-Toukei 2015 software (Social Survey Research Information Co., Ltd., Tokyo, Japan) to perform all statistical analyses.

\section{Results \\ Growth parameters}

Food intake during the experimental period did not differ among the groups (Fig. 1, Table 2). There was no significant difference among the groups in body weight during the first 3 weeks of the experiment, but after that,

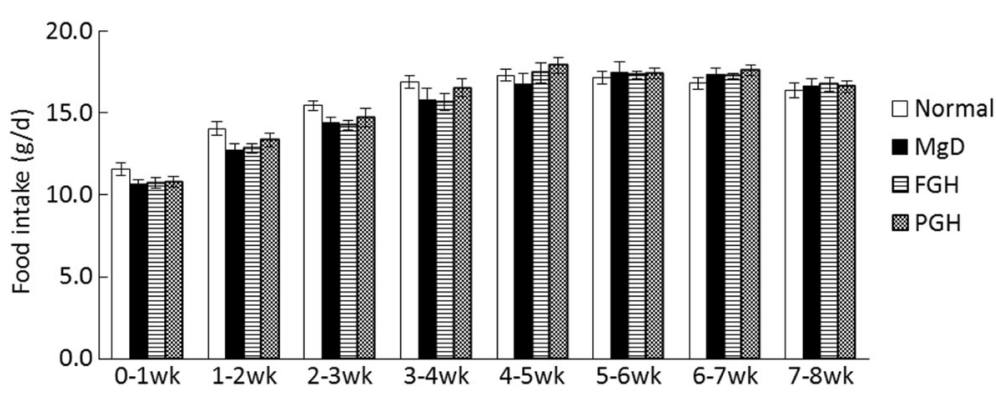

Fig. 1 Food intake. Values are presented as mean \pm SE. No significant difference among the groups was observed at all time points 
Table 2 Growth parameters

\begin{tabular}{lllll}
\hline & Normal & MgD & FGH & PGH \\
\hline $\begin{array}{l}\text { Food intake } \\
\text { ( } / \text { d })\end{array}$ & $15.9 \pm 0.2$ & $15.4 \pm 0.4$ & $15.4 \pm 0.3$ & $15.7 \pm 0.3$ \\
$\begin{array}{l}\text { Final body } \\
\text { weight (g) }\end{array}$ & $292 \pm 5^{\mathrm{a}}$ & $268 \pm 7^{\mathrm{b}}$ & $277 \pm 5^{\mathrm{a}, \mathrm{b}}$ & $277 \pm 6^{\mathrm{a}, \mathrm{b}}$ \\
$\begin{array}{l}\text { Food efficiency } \\
\text { ( } / \mathrm{g} / \mathrm{g})\end{array}$ & $0.247 \pm 0.005^{\mathrm{a}}$ & $0.227 \pm 0.002^{\mathrm{b}}$ & $0.237 \pm 0.003^{\mathrm{a}}$, b & $0.233 \pm 0.002^{\mathrm{a}, \mathrm{b}}$ \\
\hline
\end{tabular}

Values are presented as mean \pm SE. Values with different superscript letters within rows are significantly different $(p<0.05)$

body weight was significantly higher in the normal group than in the MgD group (Fig. 2). Final body weight and food efficiency were significantly lower in the MgD group than in the normal group.

\section{BMD and Ct.Th of the whole femur}

The BMD of the femur was significantly lower in the $\mathrm{MgD}$ and PGH groups than the normal group and tended to be higher $(p=0.0559)$ in the FGH group than the MgD group (Table 3). Ct.Th was significantly higher in the FGH and PGH groups than the normal group, and cortical BMD was significantly higher in the FGH group than the normal group. Trabecular BMD was significantly lower in the MgD, FGH, and PGH groups than in the normal group, but was significantly higher in the FGH group than the MgD group. The minimum sample sizes for detecting the differences between the MgD and FGH groups in the trabecular BMD and BMD at $\alpha=$ 0.05 (two-sided) and $(1-\beta)=0.8$, determined using an unpaired t-test, were 7 and 5 , respectively, which suggests that the sample size of this study seems to be statistically valid.

\section{Mechanical testing}

For the intrinsic biomechanical properties, there were no significant differences in the ultimate stress among the groups (Table 4). Young's modulus was significantly

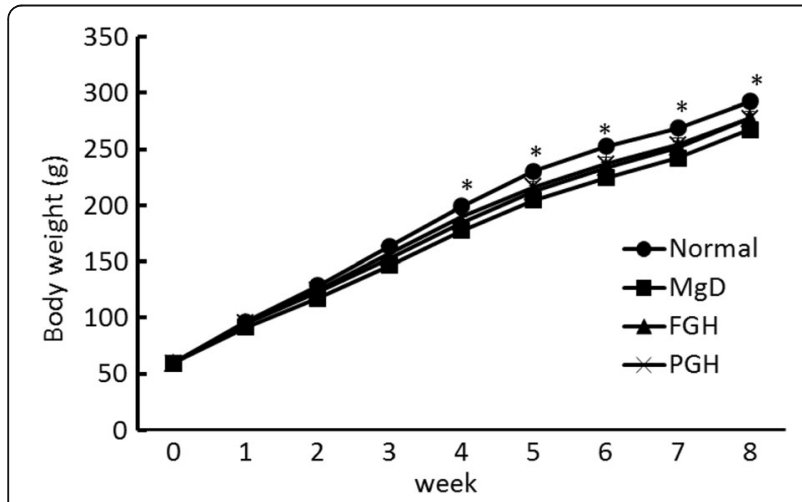

Fig. 2 Body weight. Values are presented as mean \pm SE. * $p<0.05$ vs MgD
Table 3 Bone mineral density (BMD) and cortical thickness (Ct.Th) of the whole femur

\begin{tabular}{lllll}
\hline & Normal & MgD & FGH & PGH \\
\hline BMD $\left(\mathrm{mg} / \mathrm{cm}^{3}\right)$ & $656 \pm 3^{\mathrm{a}}$ & $629 \pm 5^{\mathrm{b}}$ & $649 \pm 7^{\mathrm{a}, \mathrm{b}}$ & $633 \pm 6^{\mathrm{b}}$ \\
$\begin{array}{l}\text { Cortical BMD } \\
\left(\mathrm{mg} / \mathrm{cm}^{3}\right)\end{array}$ & $1058 \pm 4^{\mathrm{a}}$ & $1062 \pm 4^{\mathrm{a}, \mathrm{b}}$ & $1073 \pm 4^{\mathrm{b}}$ & $1070 \pm 2^{\mathrm{a}, \mathrm{b}}$ \\
$\begin{array}{l}\text { Trabecular BMD } \\
\left(\mathrm{mg} / \mathrm{cm}^{3}\right)\end{array}$ & $393 \pm 3^{\mathrm{a}}$ & $358 \pm 5^{\mathrm{c}}$ & $375 \pm 4^{\mathrm{b}}$ & $361 \pm 5^{\mathrm{b}, \mathrm{c}}$ \\
Ct.Th $(\mu \mathrm{m})$ & $415 \pm 2^{\mathrm{a}}$ & $423 \pm 4^{\mathrm{a}, \mathrm{b}}$ & $433 \pm 6^{\mathrm{b}}$ & $433 \pm 4^{\mathrm{b}}$ \\
\hline
\end{tabular}

Values are presented as mean \pm SE. Values with different superscript letters within rows are significantly different $(p<0.05)$

lower in the MgD, FGH, and PGH groups than in the normal group.

For the extrinsic biomechanical properties, there were no significant differences in the ultimate force, work to failure, and stiffness among the groups; however, ultimate force and work to failure tended to be higher ( $p=$ 0.0689, $p=0.0912$, respectively) in the PGH group than the normal group. Ultimate displacement was significantly higher in the FGH and PGH groups than the normal group.

\section{Biochemical factors}

Serum Mg levels were significantly lower in the MgD, FGH, and PGH groups than the normal group (Table 5). There were no significant differences in the serum total OC, ALP activity, TRACP5b, and CTX among the groups. The CTX/TRACP5b ratio was significantly higher in the MgD group than the normal group. There were no significant differences in the CTX/TRACP5b ratio between the normal group and the FGH and PGH groups.

\section{Discussion}

This study aimed to investigate the effects of FGH and PGH intake on BMD and intrinsic biomechanical properties in Mg-deficient rats. In the present study, a low $\mathrm{Mg}$ diet had an adverse effect on trabecular BMD but

Table 4 Intrinsic and extrinsic biomechanical properties of the femur by three-point bending test

\begin{tabular}{|c|c|c|c|c|}
\hline & Normal & MgD & $\mathrm{FGH}$ & PGH \\
\hline \multicolumn{5}{|c|}{ Intrinsic biomechanical properties } \\
\hline Ultimate stress (MPa) & $141 \pm 4$ & $134 \pm 6$ & $129 \pm 4$ & $132 \pm 1$ \\
\hline $\begin{array}{l}\text { Young's modulus } \\
(\mathrm{MPa})\end{array}$ & $1555 \pm 68^{a}$ & $1250 \pm 71^{b}$ & $1168 \pm 66^{b}$ & $1138 \pm 53^{b}$ \\
\hline \multicolumn{5}{|c|}{ Extrinsic biomechanical properties } \\
\hline Ultimate force $(\mathrm{N})$ & $108 \pm 3$ & $115 \pm 4$ & $113 \pm 3$ & $122 \pm 5$ \\
\hline $\begin{array}{l}\text { Ultimate displacement } \\
(\mathrm{mm})\end{array}$ & $1.00 \pm 0.02^{a}$ & $1.14 \pm 0.03^{a, b}$ & $1.18 \pm 0.05^{b}$ & $1.23 \pm 0.06^{b}$ \\
\hline Work to failure $(\mathrm{mJ})$ & $62.6 \pm 3.0$ & $66.3 \pm 4.3$ & $65.4 \pm 2.1$ & $76.2 \pm 5.2$ \\
\hline Stiffness (N/mm) & $81.4 \pm 1.8$ & $85.3 \pm 2.6$ & $80.4 \pm 3.1$ & $87.1 \pm 1.9$ \\
\hline
\end{tabular}

Values are presented as mean $\pm \mathrm{SE}$. Values with different superscript letters within rows are significantly different $(p<0.05)$ 
Table 5 Biochemical factors

\begin{tabular}{lllll}
\hline & Normal & MgD & FGH & PGH \\
\hline Mg $(\mathrm{mg} / \mathrm{dL})$ & $1.65 \pm 0.05^{\mathrm{a}}$ & $0.50 \pm 0.02^{\mathrm{b}}$ & $0.52 \pm 0.06^{\mathrm{b}}$ & $0.47 \pm 0.03^{\mathrm{b}}$ \\
Total OC $(\mathrm{ng} / \mathrm{mL})$ & $273 \pm 8$ & $306 \pm 19$ & $303 \pm 8$ & $308 \pm 8$ \\
ALP activity (units/ $\mu \mathrm{L})$ & $0.126 \pm 0.007$ & $0.113 \pm 0.007$ & $0.119 \pm 0.004$ & $0.110 \pm 0.004$ \\
TRACP5b (U/L) & $11.3 \pm 0.3$ & $9.7 \pm 0.8$ & $10.7 \pm 1.0$ & $11.4 \pm 1.1$ \\
CTX (ng/mL) & $37.3 \pm 1.1$ & $43.1 \pm 3.6$ & $37.3 \pm 1.3$ & $40.6 \pm 1.6$ \\
CTX/TRACP5b ratio (\% vs Normal) & $100 \pm 4^{\mathrm{a}}$ & $139 \pm 12^{\mathrm{b}}$ & $109 \pm 7^{\mathrm{a}, \mathrm{b}}$ & $113 \pm 10^{\mathrm{a}, \mathrm{b}}$ \\
\hline
\end{tabular}

Values are presented as mean \pm SE. Values with different superscript letters within rows are significantly different $(p<0.05)$

did not affect cortical BMD, which was consistent with previous reports $[25,26]$. A low $\mathrm{Mg}$ diet of $10 \%$ of the nutritional requirement was reported to increase bone resorption, while osteoblast numbers were similar to those seen in normal rats [26]. In the present study, a low $\mathrm{Mg}$ diet of $15 \%$ of the nutritional requirement increased CTX/TRACP5b ratio, known to be a useful osteoclast activity marker, but did not affect serum total OC and ALP activity, which are bone formation markers. The low Mg diet decreased Young's modulus, an intrinsic biomechanical property, which is consistent with previous data [6]. In contrast, a low Mg diet did not affect extrinsic biomechanical properties. Cortical components have a prominent role in bone strength [27], which may explain why a low Mg diet did not affect extrinsic biomechanical properties despite a decrease in trabecular BMD and intrinsic biomechanical properties. In contrast, some approximations were made in calculating bone geometry for mechanical properties, or there could be a measurement sensitivity issue with the whole bone mechanical testing, which may also explain this inconsistency.

In this study, both types of gelatin hydrolysates increased the Ct.Th and ultimate displacement, but did not prevent the decrease in the intrinsic biomechanical property. These results suggest that gelatin hydrolysates attenuate bone brittleness by increasing Ct.Th in MgD rats without affecting intrinsic biomechanical properties. Neither collagen hydrolysate prevented the decrease in serum Mg levels. Furthermore, a previous study showed that PGH intake increases the diameter of the cortical area, even in ovariectomized mice [7]. These data suggest that the beneficial effect of the gelatin hydrolysates on Ct.Th was independent of $\mathrm{Mg}$ status. In the present study, both gelatin hydrolysate groups showed a similar CTX/TRACP5b ratio as the normal group, suggesting an improvement of osteoclast activity. In addition, Kim et al. [28] reported that PGH decreases the CTX/ TRACP5b ratio in ovariectomized rats, which is consistent with our results. In the present study, the improvement of osteoclast activity may be the cause of the increase in Ct.Th.
Interestingly, only the administration of FGH prevented the decrease in trabecular BMD. Some studies reported that some oligopeptides may affect cell function and regulate gene expression in vitro [29]. Ohara et al. [16] compared quantities and structures of Hypcontaining peptides in human blood after administration of FGH and PGH and showed that the quantities and structures of peptides in human blood after oral administration of gelatin hydrolysates depend on the gelatin source, and alanine- or glycine-containing peptides were detected only in the subjects receiving FGH. An epidemiological study indicated that the intake of several amino acids, including alanine and glycine, may be beneficial for bone health [30]. Therefore, alanine- and/or glycine-containing peptides, derived from FGH, might benefit bone metabolism. Further studies are necessary to clarify the mechanism by which FGH beneficially affects trabecular BMD.

Our study has several limitations. First, we were not able to clarify whether the effects of FGH and PGH intake on BMD can be observed in other models, because we investigated the effects only in MgD rats. Second, some approximations were made in calculating bone geometry for mechanical properties, which may cause the variation. Third, there could be a measurement sensitivity issue with the whole bone mechanical testing.

\section{Conclusions}

The present study indicated that both FGH and PGH increase Ct.Th, but only FGH prevents the MgD-induced decrease in trabecular BMD seen in MgD rats, while neither type of gelatin hydrolysate affect intrinsic biomechanical properties.

\section{Abbreviations}

ALP: Alkaline phosphatase; ANOVA: Analysis of variance; BMD: Bone mineral density; CT: Computed tomography; Ct.Th: Cortical thickness; CTX: C-terminal cross-linked telopeptides of type I collagen; FGH: Fish-derived gelatin hydrolysates; Hyp: Hydroxyproline; IACUC: Institutional Animal Care and Use Committee; Mg: Magnesium; MgD: Mg-deficient; OC: Osteocalcin; PGH: Porcine skin-derived gelatin hydrolysates; RDA: Recommended daily allowance; TRACP5b: Tartrate-resistant acid phosphatase 5b 


\section{Acknowledgments}

This research did not receive any specific grant from funding agencies in the public, commercial, or not-for-profit sectors other than the fund from Meiji Co. Ltd.

\section{Funding}

This work was funded by Meiji Co., Ltd..

\section{Availability of data and materials}

The datasets used and/or analysed during the current study are available from the corresponding author on reasonable request.

\section{Authors' contributions}

TN and ST conceived and designed the experiments; TN, ST, MS, TY, HI, YS, KS, and KS performed the experiments; ST and MS analyzed the data; TN, ST, TY, HI, YS, KS, and KS wrote the paper. All authors read and approved the final manuscript.

\section{Ethics approval and consent to participate}

This study was approved by the Institutional Animal Care and Use Committee (IACUC) board of the Meiji Co., Ltd. (Ethics approval code: No. 2015_3871_0088), which was affiliated with Meiji Co., Ltd..

\section{Consent for publication}

Not applicable.

\section{Competing interests}

The authors declare that they have no competing interests.

\section{Publisher's Note}

Springer Nature remains neutral with regard to jurisdictional claims in published maps and institutional affiliations.

\section{Author details}

'Division of Research and Development, Food Science Research Laboratories, Meiji Co., Ltd., 540 Naruda, Odawara, Kanagawa 250-0862, Japan. ${ }^{2}$ Graduate School of Health and Sports Science, Juntendo University, Chiba, Japan.

${ }^{3}$ Graduate School of Medicine, Juntendo University, Tokyo, Japan.

\section{Received: 4 April 2017 Accepted: 29 August 2017}

\section{Published online: 05 September 2017}

\section{References}

1. NIH Consensus Development Panel on Osteoporosis Prevention, Diagnosis, and Therapy. Osteoporosis prevention, diagnosis, and therapy. JAMA. 2001;285:785-95

2. Young V, Garza C. Dietary reference intakes for calcium, phosphorus, magnesium, vitamin D, and fluoride. In: Institute of Medicine (US) standing committee on the scientific evaluation of dietary reference intakes. Washington, DC: National Academy Press; 1997. p. 190-249.

3. Tucker KL, Hannan MT, Chen H, Cupples LA, Wilson PW, Kiel DP. Potassium, magnesium, and fruit and vegetable intakes are associated with greater bone mineral density in elderly men and women. Am J Clin Nutr. 1999:69:727-36

4. New SA, Robins SP, Campbell MK, Martin JC, Garton MJ, Bolton-Smith C, Grubb DA, Lee SJ, Reid DM. Dietary influences on bone mass and bone metabolism: further evidence of a positive link between fruit and vegetable consumption and bone health? Am J Clin Nutr. 2000;71:142-51.

5. Rude RK, Singer FR, Gruber HE. Skeletal and hormonal effects of magnesium deficiency. J Am Coll Nutr. 2009;28:131-41.

6. Kobayashi M, Hara K, Akiyama Y. Effects of vitamin K2 (menatetrenone) and alendronate on bone mineral density and bone strength in rats fed a lowmagnesium diet. Bone. 2004;35:1136-43.

7. Guillerminet F, Beaupied H, Fabien-Soulé V, Tomé D, Benhamou CL, Roux C, Blais A. Hydrolyzed collagen improves bone metabolism and biomechanical parameters in ovariectomized mice: an in vitro and in vivo study. Bone. 2010:46:827-34. doi:10.1016/j.bone.2009.10.035.

8. Wu J, Fujioka M, Sugimoto K, Mu G, Ishimi Y. Assessment of effectiveness of oral administration of collagen peptide on bone metabolism in growing and mature rats. J Bone Miner Metab. 2004;22:547-53.
9. Koyama Y, Hirota A, Mori H, Takahara H, Kuwaba K, Kusubata M, Matsubara $Y$, Kasugai S, Itoh M, Irie S. Ingestion of gelatin has differential effect on bone mineral density and body weight in protein undernutrition. J Nutr Sci Vitaminol (Tokyo). 2001:47:84-6.

10. Nomura $Y$, Oohashi K, Watanabe M, Kasugai S. Increase in bone mineral density through oral administration of shark gelatin to ovariectomized rats. Nutrition. 2005;21:1120-6.

11. Postlethwaite $A E$, Seyer JM, Kang $A H$. Chemotactic attraction of human fibroblasts to type I, II, and III collagens and collagen-derived peptides. Proc Natl Acad Sci U S A. 1978;75:871-5.

12. Laskin DL, Kimura T, Sakakibara S, Riley DJ, Berg RA. Chemotactic activity of collagen-like polypeptides for human peripheral blood neutrophils. J Leukoc Biol. 1986:39:255-66.

13. Postlethwaite $\mathrm{AE}$, Kang $\mathrm{AH}$. Collagen-and collagen peptide-induced chemotaxis of human blood monocytes. J Exp Med. 1976;143:1299-307.

14. Kim SK, Byun HG, Park PJ, Shahidi F, Angiotensin I. Converting enzyme inhibitory peptides purified from bovine skin gelatin hydrolysate. J Agric Food Chem. 2001;49:2992-7.

15. Oshima G, Shimabukuro H, Nagasawa K. Peptide inhibitors of angiotensin Iconverting enzyme in digests of gelatin by bacterial collagenase. Biochim Biophys Acta. 1979;566:128-37.

16. Ohara H, Matsumoto H, Ito K, Iwai K, Sato K. Comparison of quantity and structures of hydroxyproline-containing peptides in human blood after oral ingestion of gelatin hydrolysates from different sources. J Agric Food Chem. 2007:55:1532-5.

17. Kawai S, Takagi Y, Kaneko S, Kurosawa T. Effect of three types of mixed anesthetic agents alternate to ketamine in mice. Exp Anim. 2011;60:481-7.

18. Turner $\mathrm{CH}$, Burr DB. Basic biomechanical measurements of bone: a tutorial. Bone. 1993;14:595-608

19. Takasugi S, Ashida K, Maruyama S, Matsukiyo Y, Kaneko T, Yamaji TA. Combination of a dairy product fermented by lactobacilli and galactooligosaccharides shows additive effects on mineral balances in growing rats with hypochlorhydria induced by a proton pump inhibitor Biol Trace Elem Res. 2013;153:309-18.

20. Takasugi S, Ashida K, Maruyama S, Komaba Y, Kaneko T, Yamaji TA. Dairy product fermented by lactobacilli cancels the adverse effects of hypochlorhydria induced by a proton pump inhibitor on bone metabolism in growing rats. Br J Nutr. 2011;106:1487-94

21. Keller TS, Spengler DM, Carter DR. Geometric, elastic, and structural properties of maturing rat femora. J Orthop Res. 1986;4:57-67.

22. Turner $\mathrm{CH}$, Akhter MP, Heaney RP. The effects of fluoridated water on bone strength. J Orthop Res. 1992;10:581-7.

23. Turner $\mathrm{CH}$. Biomechanics of bone: determinants of skeletal fragility and bone quality. Osteoporos Int. 2002;13:97-104.

24. Rissanen JP, Suominen MI, Peng Z, Halleen JM. Secreted tartrate-resistant acid phosphatase $5 b$ is a marker of osteoclast number in human osteoclast cultures and the rat ovariectomy model. Calcif Tissue Int. 2008;82:108-15.

25. Gruber HE, Rude RK, Wei L, Frausto A, Mills BG, Norton HJ. Magnesium deficiency: effect on bone mineral density in the mouse appendicular skeleton. BMC Musculoskelet Disord. 2003:4:7. doi:10.1186/1471-2474-4-7.

26. Rude RK, Gruber HE, Norton HJ, Wei LY, Frausto A, Mills BG. Bone loss induced by dietary magnesium reduction to $10 \%$ of the nutrient requirement in rats is associated with increased release of substance $P$ and tumor necrosis factor-alpha. J Nutr. 2004;134:79-85.

27. Iolascon G, Napolano R, Gioia M, Moretti A, Riccio I, Gimigliano F. The contribution of cortical and trabecular tissues to bone strength: insights from denosumab studies. Clin Cases Miner Bone Metab. 2013;10:47-51.

28. Kim HK, Kim MG, Leem KH. Osteogenic activity of collagen peptide via ERK/MAPK pathway mediated boosting of collagen synthesis and its therapeutic efficacy in osteoporotic bone by back-scattered electron imaging and microarchitecture analysis. Molecules. 2013;18:15474-89. doi:10.3390/molecules181215474.

29. Mizuno M, Kuboki Y. Osteoblast-related gene expression of bone marrow cells during the osteoblastic differentiation induced by type I collagen. J Biochem. 2001;129:133-8.

30. Jennings A, MacGregor A, Spector T, Cassidy A. Amino acid intakes are associated with bone mineral density and prevalence of low bone mass in women: evidence from discordant monozygotic twins. J Bone Miner Res. 2016:31:326-35. doi:10.1002/jbmr.2703. 